\title{
Time delays, population, and economic development
}

Luca Gori, Luca Guerrini, and Mauro Sodini

Citation: Chaos 28, 055909 (2018); doi: 10.1063/1.5024397

View online: https://doi.org/10.1063/1.5024397

View Table of Contents: http://aip.scitation.org/toc/cha/28/5

Published by the American Institute of Physics

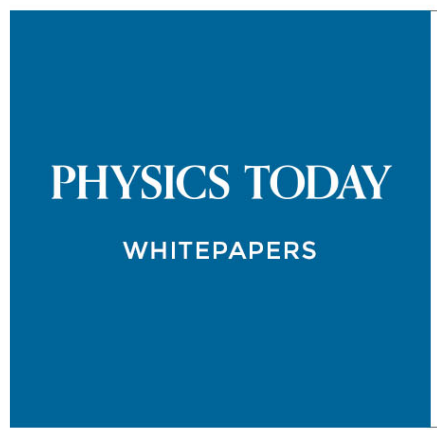

ADVANCES IN PRECISION MOTION CONTROL

Piezo Flexure Mechanisms and Air Bearings
READ NOW

PRESENTED BY

PI 


\title{
Time delays, population, and economic development
}

\author{
Luca Gori, ${ }^{1, a)}$ Luca Guerrini, ${ }^{2, b)}$ and Mauro Sodini, ${ }^{3, c)}$ \\ ${ }^{1}$ Department of Political Science, University of Genoa, Piazzale E. Brignole, 3a, I-16125 Genoa (GE), Italy \\ ${ }^{2}$ Department of Management, Polytechnic University of Marche, Piazza Martelli 8, 60121 Ancona (AN), Italy \\ ${ }^{3}$ Department of Economics and Management, University of Pisa, Via Cosimo Ridolfi, 10, I-56124 Pisa (PI), Italy
}

(Received 31 January 2018; accepted 6 April 2018; published online 22 May 2018)

\begin{abstract}
This research develops an augmented Solow model with population dynamics and time delays. The model produces either a single stationary state or multiple stationary states (able to characterise different development regimes). The existence of time delays may cause persistent fluctuations in both economic and demographic variables. In addition, the work identifies in a simple way the reasons why economics affects demographics and vice versa. Published by AIP Publishing.

https://doi.org/10.1063/1.5024397
\end{abstract}

The coevolution between economic and population dynamics is a relevant and multidisciplinary objective studied by economists, mathematicians, demographers, ecologists, and biologists. The relationships between economics and demographics have characterised both ancient and modern civilisations (Livi-Bacci, 2017) with phases where cycles were observed in both income and total population and phases where only one of these two variables fluctuated. This work aims at clarifying the links between these two variables by considering a Solowlike growth model augmented with time delays in technology and population dynamics. This allows us to account for a lag from the time the initial investment was carried out to the time it actually becomes productive and a lag from the time people were born to the time they will be economically active (belonging to the working population) and sexually active. Then, the Solow model becomes able to explain both the convergence towards a high equilibrium or a Malthusian trap and long-term fluctuations. The analysis was performed by combining stability and bifurcation results of delay differential equations and simulative exercises.

\section{INTRODUCTION}

The relationship between economic and population dynamics is a complex phenomenon that (especially in recent decades) has drawn the attention of several economists and demographers (Ehrlich and Lui, 1997; Galor, 2011, 2012; Dalgaard and Strulik, 2013; and Livi-Bacci, 2017). Since the pioneering contributions of Leibenstein (1957) and Becker (1960) until the more recent works that gave rise to the Unified Growth Theory (Galor and Weil, 2000; Galor and Moav, 2002; and 2004), which represents the economic theory of the demographic transition (DT), the link between economic and demographic variables was

\footnotetext{
${ }^{\text {a) }}$ Author to whom correspondence should be addressed: luca.gori@unige.it or dr.luca.gori@gmail.com. Tel.: +39010 20995 03. Fax: +39010 209 5536.

${ }^{b}$ E-mail: luca.guerrini@univpm.it. Tel.: +390712207055.

${ }^{c)}$ E-mail: mauro.sodini@unipi.it. Tel.: +39050 2216 234. Fax: +39050 22 10603.
}

certainly recognised to be essential to explain economic development, which is a phenomenon related-amongst other things - to fertility, mortality, migration, the environment, the distribution of income, and so on. However, it is often difficult (given the complexity of the models used) to identify the chain of causation between these variables. In addition, much of the neoclassical theory of economic growth is characterised by the results following the (standard) assumption for which population is allowed to grow without bounds [for example, the version of the Ramsey model popularised by Barro and Sala-i-Martin (2003)], although this is at odds with the historical pattern of the DT. In fact, the course of total population (that depends on fertility and mortality rates) has experienced phases of stagnation (first stage of the DT), growth (second, third, and fourth stages), and a new phase of stagnation or even decline (fifth stage). This last phase is due to the fact that mortality rates worldwide are estimated to remain low, whereas fertility rates will tend to follow a decreasing trajectory landing on below-replacement values (Fig. 1).

As is known, the dynamics of an isolated population is often described by a logistic (differential) equation characterised by a carrying capacity representing the state to which the population tends in the long term, that is, the maximum number of agents that can be sustained by the environment. Certainly, economic development can affect the carrying capacity of human population (which is driven by technological changes in a broad sense or by the accumulation of physical capital and the accumulation of human capital). From an economic point of view, although it does not consider optimising agents - as instead is usual in growth models with and without demographic variables - the pioneering contribution of Solow (1956) represents a paradigm able to describe from both theoretical and empirical points of view the long-term behaviours of countries. In this regard, we recall the different (augmented) versions of the Solow model developed over the decades that include the contribution of Mankiw et al. (1992) employing physical and human capital (as well as the subsequent empirical literature that originated from it) and the contribution of Brock and Scott Taylor (2010), formerly known as the Green Solow model, where the Solow set up is used to study environmental issues. 


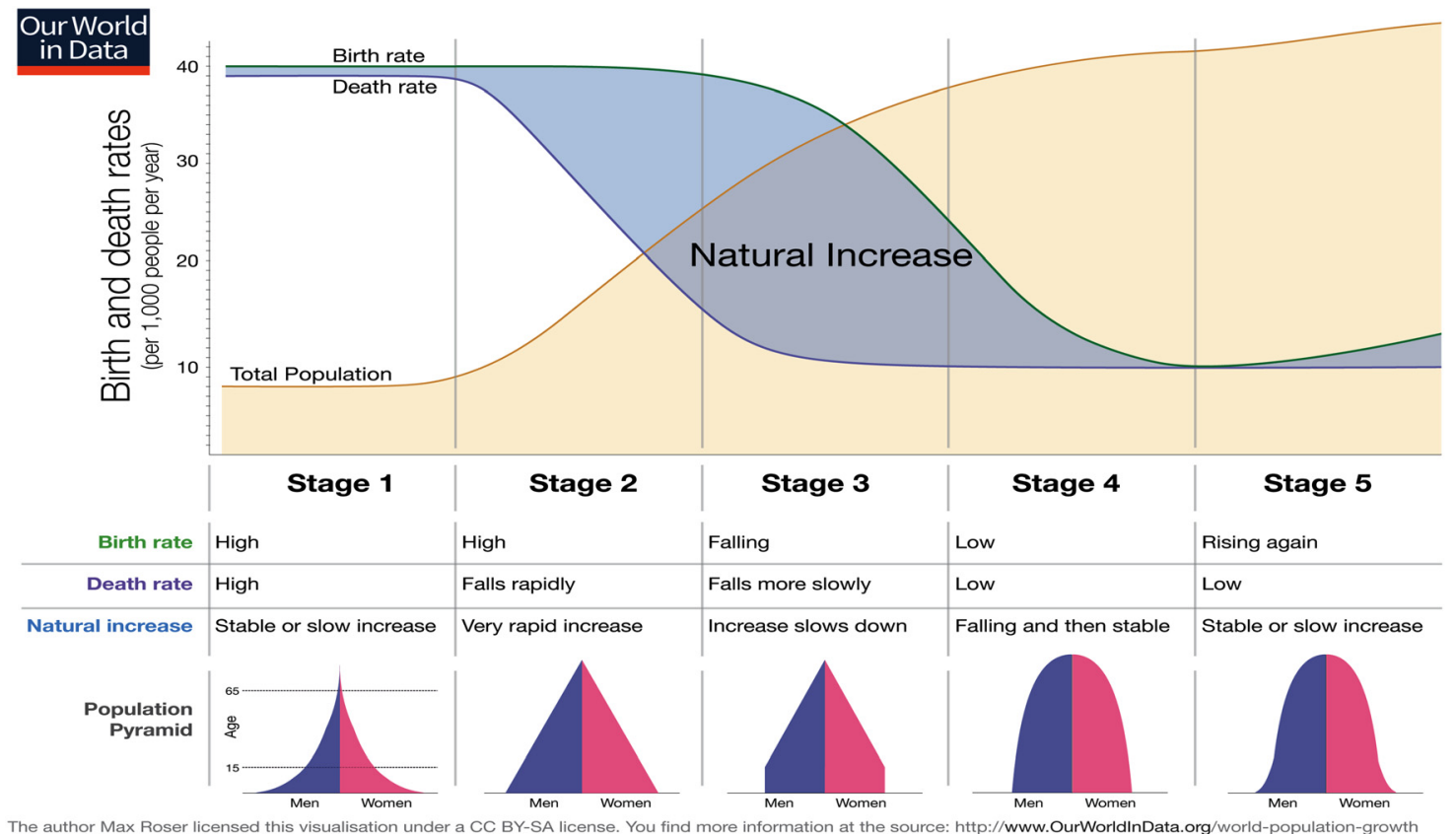

FIG. 1. Demographic transition. Reproduced with permission from M. Roser and E. Ortiz-Ospina, "World Population Growth,” 2018, see https://ourworldindata.org/world-population-growth. Copyright 2018 Creative Commons License (Roser and Ortiz-Ospina, 2018).

Recently, in two similar works, Cai (2010) and Cai (2012) augmented Solow by considering that total population evolves according to the logistic law with an endogenous carrying capacity positively correlated with the accumulation of physical capital. He studied the dynamics of the resulting system described by two ordinary differential equations that can generate either a globally attracting stationary state or three stationary states (two of which are attracting). The former case resembles Solow (1956) allowing to explain the standard convergence process of countries towards the same long-term equilibrium. The latter case instead is useful for explaining the emergence of a Malthusian trap. Therefore, converging towards an under-development regime, where income and total population are low, or a development regime, where income and total population are high, depends on the initial conditions (history matters).

A feature that, however, does not emerge in all the works cited above is represented by the possibility of capturing and describing (persistent) economic and demographic fluctuations, as instead was observed in some empirical works (Doepke et al., 2015 and Jones and Schoonbroodt, 2016). In particular, income fluctuations are a long-term phenomenon involving both advanced and developing countries. Two relevant (implicit) assumptions of this kind of models are that investments in physical capital become immediately productive (there is no lag between the investment in new capital stock and the time it is used in the production process), and the entire population can immediately give birth to children that are in turn immediately employed in a full employment setting. Some works in the literature have shown how the introduction of time delays in this framework can actually generate cycles. In particular, by considering an otherwise standard Solow model with constant population, Zak (1999) showed that the introduction of a time-to-build technology (that is, there are gestation lags in the investment process) is able to generate fluctuations (business cycles and persistent fluctuations). From a mathematical point of view, it is well known that the delayed logistic equation can undergo a Hopf bifurcation and generate attracting cycles [see Banks (1994) for details and applications in biology and demography]. Differently, Fanti and Manfredi (2003) introduced an age structure captured by distributed time delays in a Solow model where population (that was assumed to grow indefinitely) is endogenous and depends on the production activity. They showed that a demographically founded formulation of the total population may actually cause economic cycles.

The present research enters the debate about economic and demographic cycles in neoclassical growth models by augmenting Cai (2010) with a time-to-build technology and also assuming the existence of a delay from the time people were born to the time they will be economically active (belonging to the working population) and sexually active. Under these assumptions, the Solow model becomes able to identify how population cycles may cause economic cycles or vice versa. The work also provides an analysis of the interactions between cycles generated independently by the two equations of the system (i.e., when there is a one-way effect of capital dynamics on population dynamics or vice versa) as well as when there exists a double feedback between capital dynamics and population dynamics. This result is important as it helps in clarifying some transmission mechanisms between economic and demographic variables that are often difficult to identify in growth models belonging to the Unified Growth Theory because of the complexity of the mixture of influences that demographics and economics have in this class of models.

The rest of the work proceeds as follows: Section II builds on the model with fixed delays. Sections III and IV provide analytical results on the existence and stability of the 
stationary state equilibria in some particular cases. Section V introduces the technique of the stability crossing curves developed by Lin and Wang (2012) to analyse the local stability properties of the dynamic system. In addition, it provides some insights (through simulative exercises) on model dynamics when the equilibria turn out to be unstable. Section VI outlines the conclusions.

\section{THE MODEL}

In a recent work, Cai (2010) has studied a Solow-type growth model augmented with carrying capacity of human population, which is assumed to depend on capital accumulation. Capital accumulation in fact can be considered as a proxy of economic growth and development that can move the carrying capacity forward. This has allowed the author to overcome the standard assumption of the growth literature for which population is assumed to grow without bounds ( $\dot{L} / L=n>0)$, which is, however, not empirically well supported (see the Introduction for a discussion on this issue). His model is summarised here in system (1)

$$
\left\{\begin{array}{l}
\dot{K}=s A K^{\alpha} L^{1-\alpha}-\delta K, \\
\dot{L}=\gamma L\left[1-\frac{L}{g(K)}\right],
\end{array}\right.
$$

where $K$ is capital, $L$ denotes $s \in(0,1)$ is the fixed saving rate, $A>0$ is the exogenous total factor productivity, $\alpha \in(0,1)$ is the output elasticity of capital, $\delta \in(0,1)$ is the depreciation rate, and $\gamma>0$ measures the growth coefficient of the population. Function $g$ represents (a logistic-type) capital-dependent carrying capacity for the population and it is specified in the following way:

$$
g(K)=\frac{\bar{L}}{1+\left(\bar{L} / L_{0}-1\right) e^{-c K}},
$$

where $L_{0}$ is the initial population, $\bar{L}>L_{0}$ is the final carrying capacity, and $c>0$ is the growth coefficient of the carrying capacity. The first expression of system (1) is the standard equation of Solow (1956) that drives capital accumulation over time, which employs a Cobb-Douglas production function

$$
Y=A K^{\alpha} L^{1-\alpha} .
$$

The second equation instead describes the dynamics of human population through a modified logistic equation, which comes from assuming the population growth rate $\dot{L} / L$ be dependent negatively on the size of the population (as is standard in biological models) and positively on the level of capital accumulation. This last assumption tells us that the constraint given by the natural carrying capacity is relaxed by technological changes in a broad sense.

The augmented Solow model of Cai (2010) allows to have a theoretical framework able to explain the existence of long-term stationary states representing two relevant paradigms, that is, sustained development on one hand and Malthusian trap on the other hand. However, the model of Cai cannot explain some features that are instead relevant in growth models that explicitly include demographic variables. In order to overcome this lacuna, we have revisited Cai (2010) by assuming the existence of a time-to-build technology with respect to which the use of capital in production requires time. This implies that investments can effectively be employed by the existing technology as a capital input in production only with a time delay $\tau_{1} \geq 0$ (Zak, 1999). In addition, there exists a delay $\tau_{2} \geq 0$ from the time people were born to the time they will become economically active (belonging to the working population) and sexually active. Then, the growth rate of population $\dot{L} / L$ depends on both the capital stock currently used in production (which was installed at time $t-\tau_{1}$ ) and economically and sexually active individuals (born at time $t-\tau_{2}$ ). As will be shown later in this article, this version of the (augmented) Solow model with endogenous population and time delays will allow explaining economic development and persistent fluctuations. It is also able to identify the chain of causation and the interplay between economic and demographic variables.

Given the assumptions discussed above, system (1) modifies to become the following:

$$
\left\{\begin{array}{l}
\dot{K}=s A K_{\tau_{1}}^{\alpha} L_{\tau_{2}}^{1-\alpha}-\delta K_{\tau_{1}}, \\
\dot{L}=\gamma L\left[1-\frac{L_{\tau_{2}}}{g\left(K_{\tau_{1}}\right)}\right],
\end{array}\right.
$$

where $K_{\tau_{1}}:=K\left(t-\tau_{1}\right)$ and $L_{\tau_{2}}:=L\left(t-\tau_{2}\right)$.

Equilibria or steady states of system (4) coincide with the corresponding steady states for the case of zero delays. From Cai (2010), we know that there exist either one or three non-trivial equilibria $\left(K^{*}, L^{*}\right)$ such that

$$
s A\left(K^{*}\right)^{\alpha-1}\left(L^{*}\right)^{1-\alpha}=\delta \text { and } g\left(K^{*}\right)=L^{*} .
$$

As regards the model without delays $\left(\tau_{1}=\tau_{2}=0\right)$, it is easy to check that when the steady state equilibrium is unique it is globally asymptotically stable. This implies that a generic trajectory generated by any positive couples $(K, L)$ of initial conditions will converge towards the steady state [this is in line with the stylised fact of Solow's convergence, as pointed out in Mankiw et al. (1992)]. Differently, when there exist three steady states $\left(K_{1}^{*}, L_{1}^{*}\right),\left(K_{2}^{*}, L_{2}^{*}\right)$, and $\left(K_{3}^{*}, L_{3}^{*}\right)$ such that $K_{1}^{*}<K_{2}^{*}<K_{3}^{*}$ and $L_{1}^{*}<L_{2}^{*}<L_{3}^{*}$, the first and the third equilibriums are attracting, whereas the second one is a saddle whose stable manifold separates the basins of attraction of the two attractors. In this case, the model is able to describe the coexistence of a high equilibrium capturing the paradigm of developed countries and a low equilibrium capturing the paradigm of developing or under-developed countries (Malthusian trap). Converging towards $\left(K_{1}^{*}, L_{1}^{*}\right)$ or $\left(K_{3}^{*}, L_{3}^{*}\right)$ is a matter of initial conditions. This result is in line with empirical evidence about the existence of multiple growth paths and different convergence groups of countries with distinct values of income and population growth rates (Palivos, 1995).

In order to study the stability of equilibria of system (4), we consider the characteristic equation of the linearisation of (4) at a generic equilibrium $\left(K^{*}, L^{*}\right)$ and get 


$$
\lambda^{2}+a \lambda e^{-\lambda \tau_{1}}+\gamma \lambda e^{-\lambda \tau_{2}}+a \gamma\left(1-M_{i}\right) e^{-\lambda\left(\tau_{1}+\tau_{2}\right)}=0,
$$

where

$$
a=(1-\alpha) \delta>0, M_{i}=g_{K}^{\prime}\left(K_{i}^{*}\right) \frac{K_{i}^{*}}{L_{i}^{*}}>0, \text { with } i=\{1,2,3\} .
$$

In the case of no delays $\left(\tau_{1}=0\right.$ and $\left.\tau_{2}=0\right)$, Cai (2010) (Theorem 2, p. 3466) showed that a positive equilibrium point $\left(K^{*}, L^{*}\right)$ of system (4) is asymptotically stable if $M_{i}<1$ (it is a sink), it is unstable if $M_{i}>1$ (it is a saddle), and nonhyperbolic if $M_{i}=1$. In what follows, we assume that $M_{i} \neq 1$.

\section{CASE $\tau_{1}=0, \tau_{2}>0$}

The characteristic equation (5) becomes

$$
\lambda^{2}+a \lambda+\left[a \gamma\left(1-M_{i}\right)+\gamma \lambda\right] e^{-\lambda \tau_{2}}=0 .
$$

We first note that Eq. (7) has no zero root being $M_{i} \neq 1$. Let $\lambda=i \omega(\omega>0)$ be a root of (7). Then, plugging it into (7), and separating the real and the imaginary parts, yields

$$
\begin{aligned}
& \omega^{2}=a \gamma\left(1-M_{i}\right) \cos \omega \tau_{2}+\gamma \omega \sin \omega \tau_{2}, \\
& a \omega=-\gamma \omega \cos \omega \tau_{2}+a \gamma\left(1-M_{i}\right) \sin \omega \tau_{2} .
\end{aligned}
$$

Squaring both sides and adding them up lead to the following polynomial equation in $\omega^{2}$ :

$$
\omega^{4}+\left(a^{2}-\gamma^{2}\right) \omega^{2}-\left[a \gamma\left(1-M_{i}\right)\right]^{2}=0 .
$$

It is clear that Eq. (9) has a positive root $\omega_{+}$. Furthermore, solving Eq. (8) for $\tau_{2}$ gives

$$
\begin{aligned}
\sin \omega \tau_{2} & =\frac{\left[1+a^{2}\left(1-M_{i}\right)\right] \omega}{\gamma \omega^{2}+a^{2} \gamma\left(1-M_{i}\right)^{2}}, \\
\cos \omega \tau_{2} & =-\frac{a M_{i} \omega^{2}}{\gamma \omega^{2}+a^{2} \gamma\left(1-M_{i}\right)^{2}} .
\end{aligned}
$$

Hence, we can derive the values of $\tau_{2}$ at which there exists the root $i \omega_{+}$of (7).

In conclusion, we have the following result.

Lemma 1. Let $a$ and $M_{i}$ be defined as in (6). There exists a sequence of positive numbers $\tau_{2, j}^{(+)}(j=0,1,2, \ldots)$ such that $0<\tau_{2,0}^{(+)}<\tau_{2,1}^{(+)}<\cdots<\tau_{2, j}^{(+)}<\cdots$, and (7) has a pair of purely imaginary roots $\pm \mathrm{i} \omega_{+}$when $\tau_{2}=\tau_{2, j}^{(+)}$, where

$$
\omega_{+}=\sqrt{\frac{-\left(a^{2}-\gamma^{2}\right)+\sqrt{\left(a^{2}-\gamma^{2}\right)^{2}+4\left[a \gamma\left(1-M_{i}\right)\right]^{2}}}{2}}
$$

and

$$
\tau_{2, j}^{(+)}=\left\{\begin{array}{l}
\frac{1}{\omega_{+}} \cos ^{-1}\left\{-\frac{a M_{i} \omega^{2}}{\gamma \omega^{2}+a^{2} \gamma\left(1-M_{i}\right)^{2}}\right\}+\frac{2 j \pi}{\omega_{+}}, \quad \text { if } 1+a^{2}\left(1-M_{i}\right) \geq 0, \\
\frac{2(j+1) \pi}{\omega_{+}}-\frac{1}{\omega_{+}} \cos ^{-1}\left\{-\frac{a M_{i} \omega^{2}}{\gamma \omega^{2}+a^{2} \gamma\left(1-M_{i}\right)^{2}}\right\}, \quad \text { if } 1+a^{2}\left(1-M_{i}\right)<0 .
\end{array}\right.
$$

Let $\lambda\left(\tau_{2}\right)$ be the root of (7) such that $\operatorname{Re}\left(\tau_{2, j}^{(+)}\right)=0, \operatorname{Im}\left(\tau_{2, j}^{(+)}\right)=\omega_{+}$. The variation direction of its real part with respect to the time delay $\tau_{2}$ can be studied through the sign of $\left[d(\operatorname{Re} \lambda) / d \tau_{2}\right]_{\tau_{2}=\tau_{2}^{(+)}}$. The next result shows that each crossing of the roots through the imaginary axis in the complex plane occurs from left to right

Proposition 2. $\lambda= \pm \mathrm{i} \omega_{+}$are simple roots of (7) at $\tau_{2}=\tau_{2, j}^{(+)}$and

$$
\left[\frac{d(\operatorname{Re} \lambda)}{d \tau_{2}}\right]_{\tau_{2}=\tau_{2, j}^{(+)}}>0
$$

Proof. Differentiating Eq. (7) with respect to $\tau_{2}$, we get

$$
\begin{aligned}
& \left\{2 \lambda+a+\gamma e^{-\lambda \tau_{2}}-\left[a \gamma\left(1-M_{i}\right)+\gamma \lambda\right] \tau_{2} e^{-\lambda \tau_{2}}\right\}\left(\frac{d \lambda}{d \tau_{2}}\right) \\
& =\left[a \gamma\left(1-M_{i}\right)+\gamma \lambda\right] e^{-\lambda \tau_{2}} .
\end{aligned}
$$

Notice that $i \omega_{+}$must be a simple root of (7), otherwise, from (11), $\left[a \gamma\left(1-M_{i}\right)+\gamma i \omega_{+}\right] e^{-i \omega_{+} \tau_{2, j}^{(+)}}=0$, i.e., the absurd $\omega_{+}=0$. Then, we have

$$
\left(\frac{d \lambda}{d \tau_{2}}\right)^{-1}=\frac{(2 \lambda+a) e^{\lambda \tau_{2}}+\gamma}{a \gamma\left(1-M_{i}\right)+\gamma \lambda}-\frac{\tau_{2}}{\lambda}
$$

which implies

$$
\operatorname{sign}\left[\frac{d(\operatorname{Re} \lambda)}{d \tau_{2}}\right]_{\lambda=i \omega_{+}}=\operatorname{sign}\left[\operatorname{Re}\left(\frac{d \lambda}{d \tau_{2}}\right)^{-1}\right]_{\lambda=i \omega_{+}}=\operatorname{sign}\left\{\gamma^{2}-a^{2}+2 \omega_{+}^{2}\right\}=\operatorname{sign}\left\{\sqrt{\left(a^{2}-\gamma^{2}\right)^{2}+4\left[a \gamma\left(1-M_{i}\right)\right]^{2}}\right\} .
$$

This completes the proof. 
Based on the above transversality condition and the Hopf bifurcation theorem, one has the following results.

Theorem 3. Let $\tau_{2,0}^{(+)}$be defined as in (10).

(1) If $M_{i}>1$, then system (4) is unstable for any given time delay $\tau_{2}$.

(2) If $M_{i}<1$, then there exists exactly one critical time delay $\tau_{2,0}^{(+)}>0$ such that the system remains locally asymptotically stable when $0 \leq \tau_{2}<\tau_{2,0}^{(+)}$and becomes unstable when $\tau_{2}>\tau_{2,0}^{(+)}$. System (4) undergoes a Hopf bifurcation at $\left(K^{*}, L^{*}\right)$ for $\tau_{2}=\tau_{2,0}^{(+)}$.

\section{CASE $\tau_{1}>0, \tau_{2}$ FIXED}

We now let $\tau_{2}$ be fixed at a non-negative given value and assume $\tau_{1}$ be a bifurcation parameter. Let $\lambda=i \omega(\omega>0)$ be a root of (5), i.e.,

$$
-\omega^{2}+a i \omega e^{-i \omega \tau_{1}}+\gamma i \omega e^{-i \omega \tau_{2}}+a \gamma\left(1-M_{i}\right) e^{-i \omega\left(\tau_{1}+\tau_{2}\right)}=0 .
$$

Separating real and imaginary parts leads to

$$
\begin{aligned}
-\omega^{2}+\gamma \omega \sin \omega \tau_{2}= & {\left.\left[a \gamma\left(1-M_{i}\right) \sin \omega \tau_{2}-a \omega\right]\right) } \\
& \times \sin \omega \tau_{1}-a \gamma\left(1-M_{i}\right) \cos \omega \tau_{1} \cos \omega \tau_{2},
\end{aligned}
$$

$$
\begin{aligned}
\gamma \omega \cos \omega \tau_{2}= & {\left.\left[a \gamma\left(1-M_{i}\right) \sin \omega \tau_{2}-a \omega\right]\right) \cos \omega \tau_{1} } \\
& +a \gamma\left(1-M_{i}\right) \sin \omega \tau_{1} \cos \omega \tau_{2} .
\end{aligned}
$$

Equations (12) and (13) are simplified to give

$$
\begin{aligned}
G(\omega)= & \omega^{4}+\left(-2 \gamma \sin \omega \tau_{2}\right) \omega^{3}+\left(\gamma^{2}-a^{2}\right) \omega^{2} \\
& +\left[2 a^{2} \gamma\left(1-M_{i}\right) \sin \omega \tau_{2}\right] \omega-a^{2} \gamma^{2}\left(1-M_{i}\right)^{2}=0 .
\end{aligned}
$$

Noticing that $G(+\infty)=+\infty$ and $G(0)=-a^{2} \gamma^{2}\left(1-M_{i}\right)^{2}<0$, we can conclude that Eq. (14) has at least one positive solution. Let $\omega_{1}, \omega_{2}, \ldots, \omega_{N}$ denote the positive roots of (14). For every $\omega_{k}(k=1,2, \ldots, N)$, solving Eqs. (12) and (13) for $\tau_{1}$, one can determine the corresponding critical value $\tau_{1, k}^{(j)}(j=0,1,2, \ldots)$ of $\tau_{1}$. By setting

$$
\tau_{1}^{*}=\min \left\{\tau_{1, k}^{(j)}, k=1,2, \ldots, N, j=0,1,2, \ldots\right\},
$$

then Eq. (5) has a pair of purely imaginary roots $\lambda= \pm i \omega_{*}$ at $\tau_{1}=\tau_{1}^{*}$. To verify the transversality condition of Hopf bifurcation, taking the derivative with respect to $\tau_{1}$ in (5), it is obtained that

$$
\begin{aligned}
\operatorname{sign}\left[\frac{d(\operatorname{Re} \lambda)}{d \tau_{1}}\right]_{\tau_{1}=\tau_{1}^{*}} & =\operatorname{sign}\left[\operatorname{Re}\left(\frac{d \lambda}{d \tau_{1}}\right)^{-1}\right]_{\tau_{1}=\tau_{1}^{*}} \\
& =\operatorname{sign}\left(H\left(\omega^{*}, \tau_{1}^{*}\right)\right),
\end{aligned}
$$

with

$$
H\left(\omega^{*}, \tau_{1}^{*}\right)=A B+C D,
$$

where

$$
\begin{gathered}
A=-a \omega^{*} \tau_{1}^{*} \sin \omega^{*} \tau_{1}^{*}+a \cos \omega^{*} \tau_{1}^{*} \\
+\left[-\gamma \omega^{*} \tau_{2}+a \gamma\left(1-M_{i}\right)\left(\tau_{1}^{*}+\tau_{2}\right) \sin \omega^{*} \tau_{1}^{*}\right] \sin \omega^{*} \tau_{2} \\
+\left[\gamma-a \gamma\left(1-M_{i}\right)\left(\tau_{1}^{*}+\tau_{2}\right) \cos \omega^{*} \tau_{1}^{*}\right] \cos \omega^{*} \tau_{2}, \\
B=\left\{\left[a \gamma\left(1-M_{i}\right) \sin \omega^{*} \tau_{2}-a \omega^{*}\right] \cos \omega^{*} \tau_{1}^{*}\right. \\
\left.+a \gamma\left(1-M_{i}\right) \sin \omega^{*} \tau_{1}^{*} \cos \omega^{*} \tau_{2}\right\} \omega^{*}, \\
C=\omega^{*}\left(a \tau_{1}^{*} \cos \omega^{*} \tau_{1}^{*}-2\right)+a \sin \omega^{*} \tau_{1}^{*} \\
+\left[\gamma \omega^{*} \tau_{2}-a \gamma\left(1-M_{i}\right)\left(\tau_{1}^{*}+\tau_{2}\right) \sin \omega^{*} \tau_{1}^{*}\right] \cos \omega^{*} \tau_{2} \\
+\left[\gamma-a \gamma\left(1-M_{i}\right)\left(\tau_{1}^{*}+\tau_{2}\right) \cos \omega^{*} \tau_{1}^{*}\right] \sin \omega^{*} \tau_{2}, \\
D=\left\{\left[a \gamma\left(1-M_{i}\right) \sin \omega^{*} \tau_{2}-a \omega^{*}\right] \sin \omega^{*} \tau_{1}^{*}\right. \\
\left.-a \gamma\left(1-M_{i}\right) \cos \omega^{*} \tau_{1}^{*} \cos \omega^{*} \tau_{2}\right\} \omega^{*} .
\end{gathered}
$$

A positive sign of $H\left(\omega^{*}, \tau_{1}^{*}\right)$ corresponds to crossings of the imaginary axis from right to left as $\tau_{1}$ increases, while a negative sign of $H\left(\omega^{*}, \tau_{1}^{*}\right)$ means crossings of the imaginary axis from left to right.

According to the previous analysis, we get the following conclusions.

Theorem 4. Let $G(\omega), \tau_{1}^{*}$ and $H\left(\omega^{*}, \tau_{1}^{*}\right)$ be defined as in (14), (15), and (16), respectively.

(1) If $G(\omega)$ has only one simple positive root, then

(a) if system (4) without time delays is locally asymptotically stable (resp. unstable) and $H\left(\omega^{*}, \tau_{1}^{*}\right)<0$ (resp. $H\left(\omega^{*}, \tau_{1}^{*}\right)>0$ ), then it remains stable (resp. unstable) for $\tau_{1} \geq 0$;

(b) if system (4) without time delays is locally asymptotically stable (resp. unstable) and $H\left(\omega^{*}, \tau_{1}^{*}\right)>0$ (resp. $\left.H\left(\omega^{*}, \tau_{1}^{*}\right)<0\right)$, then system (4) is stable for $\tau_{1} \in\left[0, \tau_{1}^{*}\right)$ and the equilibrium point $\left(K^{*}, L^{*}\right)$ loses (resp. acquires) its stability via a Hopf bifurcation at $\tau_{1}=\tau_{1}^{*}$.

(2) If $G(\omega)$ has at least two positive roots and these roots are simple, then a finite number of stability switches may occur as the time delay $\tau_{1}$ increases from zero to the positive infinity, with the occurrence of a Hopf bifurcation at each switch.

\section{STABILITY CROSSING CURVES AND SIMULATIVE EXERCISES}

In order to characterise the stability properties of the equilibria $\left(K_{1}^{*}, L_{1}^{*}\right)$ and $\left(K_{3}^{*}, L_{3}^{*}\right)$, we will use the stability crossing curves technique allowing to geometrically analyse the problem in the plane $\left(\tau_{1}, \tau_{2}\right)$. By following Lin and Wang (2012), we now introduce the polynomials

$$
\begin{gathered}
P_{0}(\lambda):=\lambda^{2}, \\
P_{1}(\lambda):=a \lambda, \\
P_{2}(\lambda):=\gamma \lambda, \\
P_{3}(\lambda):=a \gamma\left(1-M_{i}\right),
\end{gathered}
$$

and the function 


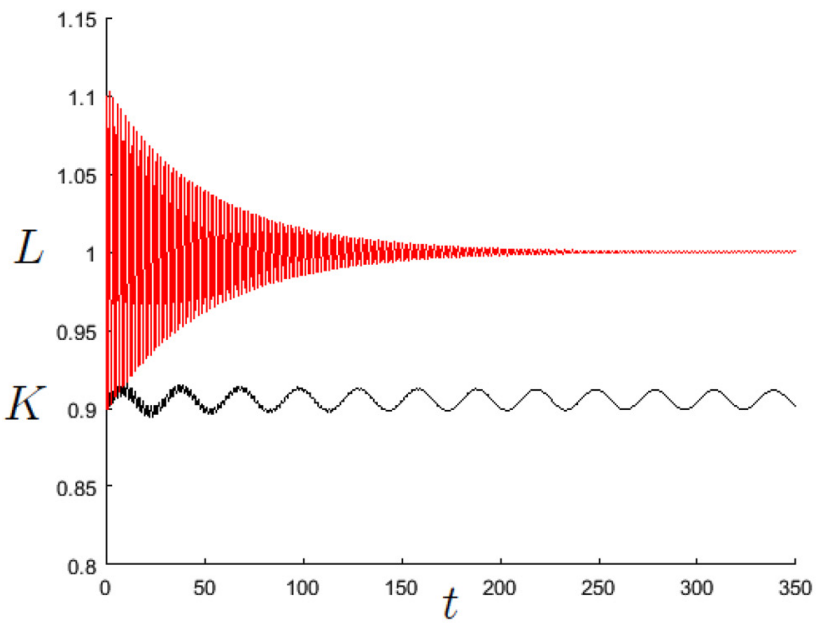

(a)

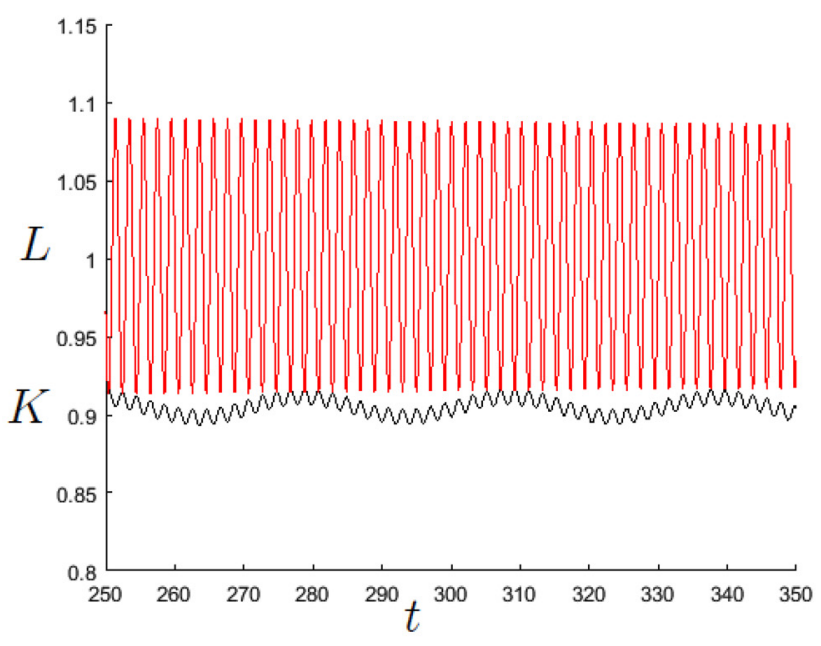

(c)

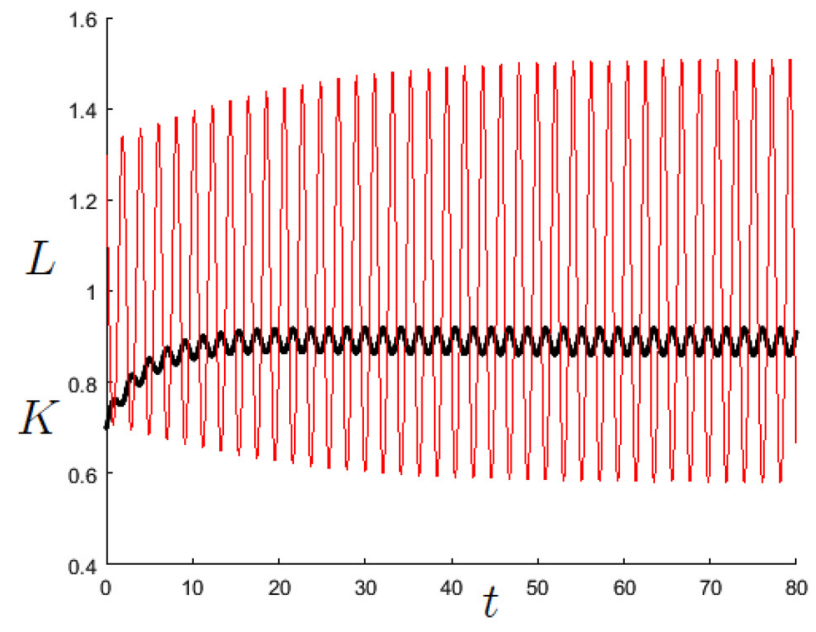

(b)

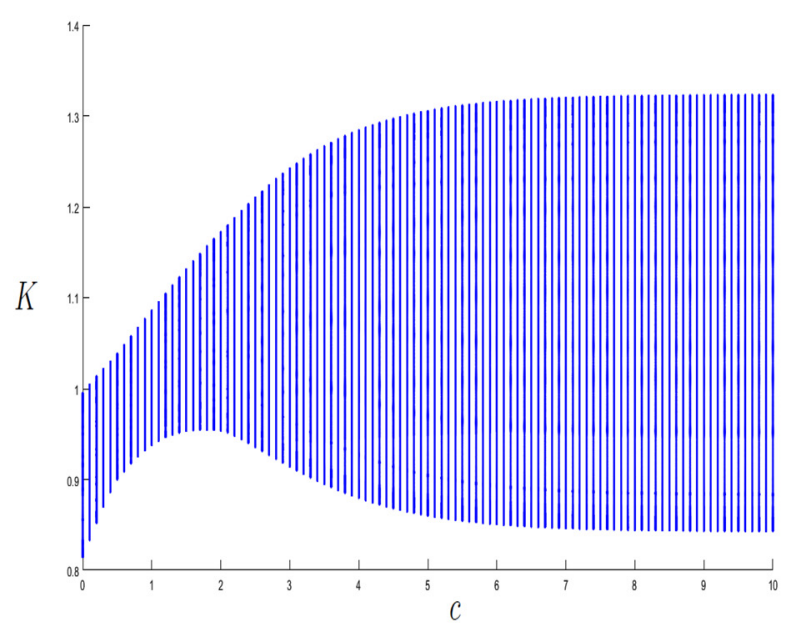

(d)

FIG. 2. Time series of $K$ (black coloured) and $L$ (red coloured). (a) Case $c=0$ and $L \rightarrow L^{*}\left(\tau_{1}=7.5\right.$ and $\left.\tau_{2}=0.48\right)$. (b) Case $c=0$ and $\tau_{1}=0$ ( $\left.\tau_{2}=0.52\right)$. (c) Case $c=0, \tau_{1}=7.5$, and $\tau_{2}=0.507$. (d) Bifurcation diagram with bifurcation parameter $c$ depicting the evolution of the attractor. We note that $c$ does not qualitatively affects the dynamics of the system.

$$
\begin{aligned}
Z(\omega):= & \left(\left|P_{0}(i \omega)\right|^{2}+\left|P_{1}(i \omega)\right|^{2}-\left|P_{2}(i \omega)\right|^{2}-\left|P_{3}(i \omega)\right|^{2}\right)^{2} \\
& -4\left(L_{1}(\omega)^{2}+L_{2}(\omega)^{2}\right),
\end{aligned}
$$

defined on $W:=\{\omega \in \mathbb{R}: \omega>0\}$, where

$$
L_{1}(\omega):=\operatorname{Re}\left(P_{2}(i \omega) \overline{P_{3}(i \omega)}\right)-\operatorname{Re}\left(P_{0}(i \omega) \overline{P_{1}(i \omega)}\right),
$$

and

$$
L_{2}(\omega):=\operatorname{Re}\left(P_{1}(i \omega) \overline{P_{3}(i \omega)}\right)-\operatorname{Re}\left(P_{0}(i \omega) \overline{P_{2}(i \omega)}\right) .
$$

As assumptions of Lin and Wang (2012, p. 521) (i) $\operatorname{deg}\left(P_{0}(\lambda)\right)=2 \geq \operatorname{maxdeg}\left\{P_{1}(\lambda), P_{2}(\lambda), P_{3}(\lambda)\right\}=1$, (ii) $P_{0}(0)+P_{1}(0)+P_{2}(0)+P_{3}(0)=a \gamma\left(1-M_{i}\right) \neq 0, \quad$ (iii) $P_{0}(\lambda), P_{1}(\lambda), P_{2}(\lambda)$, and $P_{3}(\lambda)$ are coprime polynomials and (iv)

$$
\lim _{\lambda \rightarrow \infty}\left(\left|\frac{P_{1}(\lambda)}{P_{0}(\lambda)}\right|+\left|\frac{P_{2}(\lambda)}{P_{0}(\lambda)}\right|+\left|\frac{P_{3}(\lambda)}{P_{0}(\lambda)}\right|\right)=0<1
$$

are fulfilled, it is possible to identify the $\omega$-intervals such that $Z(\omega)$ is negative and the corresponding stability crossing curves. In our case, $Z(\omega)$ reads as follows:

$$
\begin{aligned}
Z(\omega):= & \omega^{8}-2\left(a^{2}+\gamma^{2}\right) \omega^{6}+\left[\gamma^{4}-2 a^{2}\left(M_{i}+2 M_{i}-2\right) \gamma^{2}+a^{4}\right] \\
& \times \omega^{4}-2 a^{2} \gamma^{2}\left(1-M_{i}\right)^{2}\left(a^{2}+\gamma^{2}\right) \omega^{2}+a^{4} \gamma^{4}\left(1-M_{i}\right)^{4} .
\end{aligned}
$$

Given the particular form of polynomial (21), it can be factorized to obtain analytical solutions (as a function of the state variables $K$ and $L$ that can be obtained only numerically) of $Z(\omega)<0$ on set $W$ [see, for instance, Pecora and Sodini (2018)]. In what follows, we will consider two different parameter sets for which there exists either a unique steady-state equilibrium or multiple steady-state equilibria.

\section{A. Unique steady-state equilibrium}

By using the parameter values $s=0.3, A=1, \alpha=0.35$, $\delta=0.32, L_{0}=1, \bar{L}=1.2$, and $\gamma=3.1$, it is possible to verify 


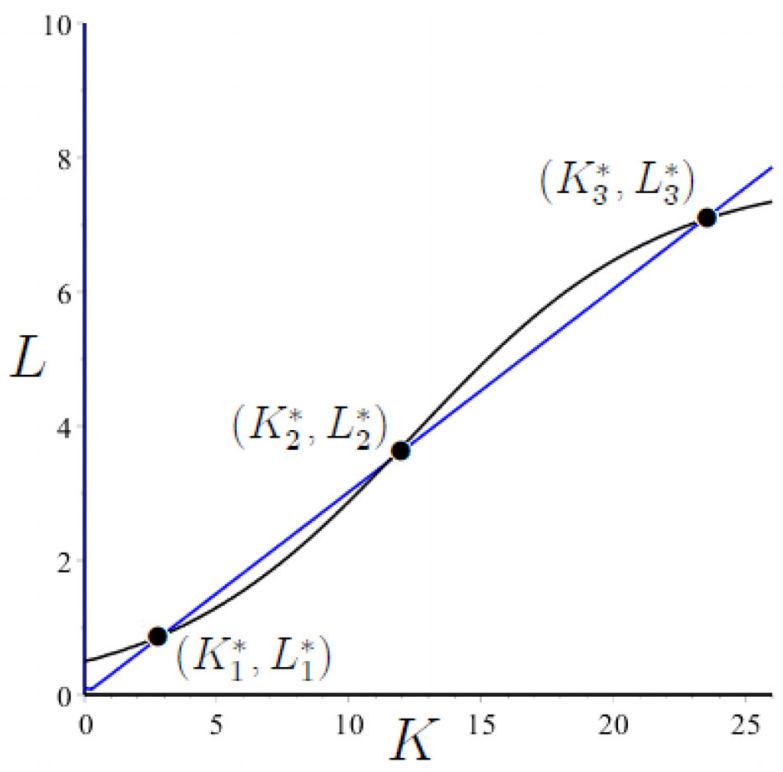

(a)

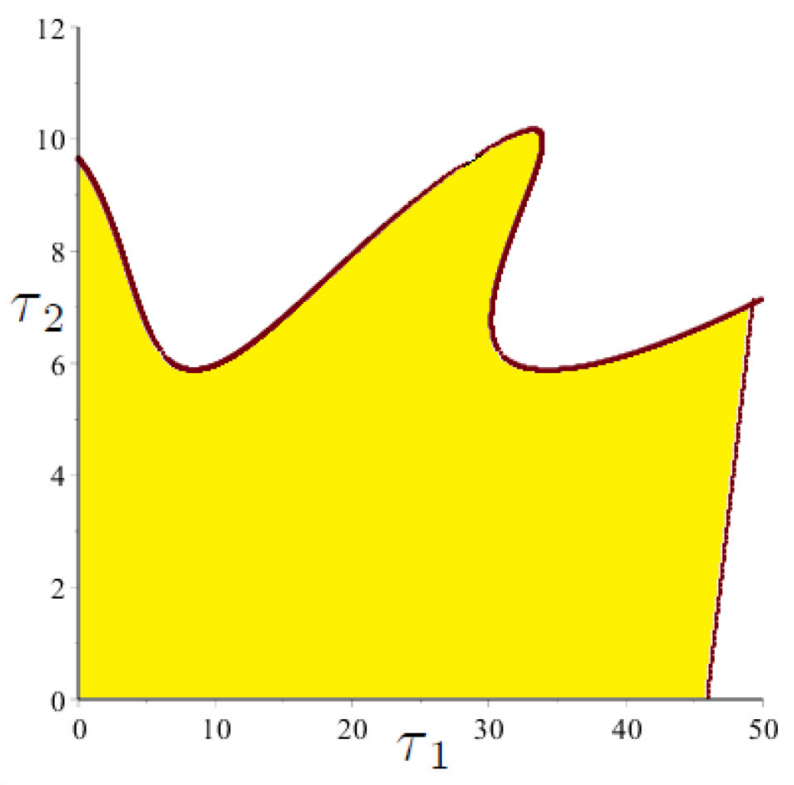

(b)

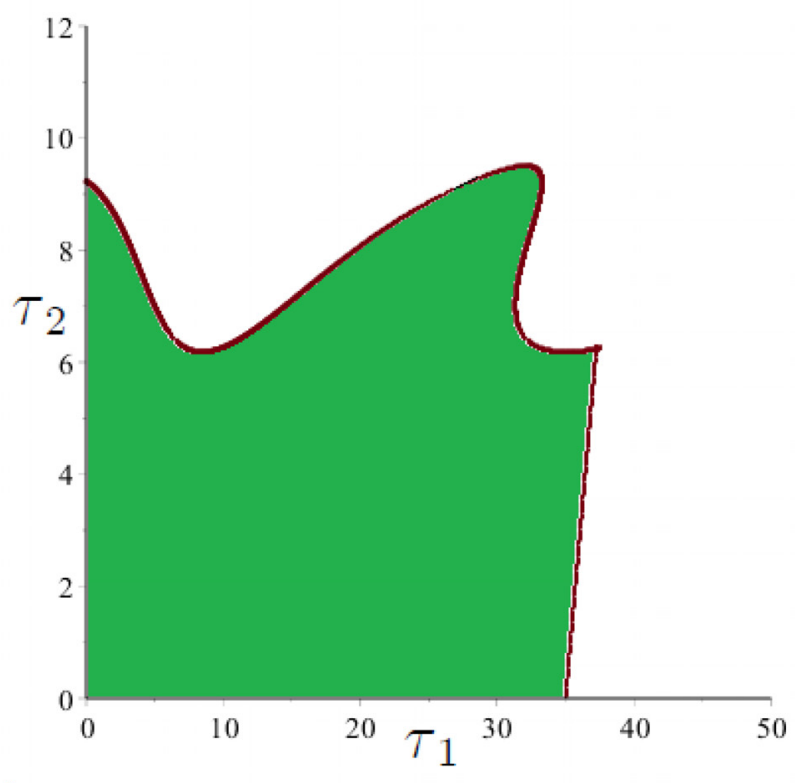

(c)

FIG. 3. (a) Stationary states in $(K, L)$ plane. Equilibria are $\left(K_{1}^{*}, L_{1}^{*}\right)=(2.95,0.89),\left(K_{2}^{*}, L_{2}^{*}\right)=(11.42,3.45)$, and $\left(K_{3}^{*}, L_{3}^{*}\right)=(23.39,7.07)$. (b) Stability crossing curves in $\left(\tau_{1}, \tau_{2}\right)$ plane related to $\left(K_{1}^{*}, L_{1}^{*}\right)$. (c) Stability crossing curves in $\left(\tau_{1}, \tau_{2}\right)$ plane related to $\left(K_{3}^{*}, L_{3}^{*}\right)$.

that the system admits a unique stationary state. In particular, as can be seen by looking at Eq. (4), the value of $L$ enters the dynamics of capital accumulation through the production function given by Eq. (3), whereas the value of $K$ enters the dynamics of total population through function $g$ specified in (2). We will now present numerical examples by distinguishing between two cases, that is, $c=0$ and $c>0$ in (2). When $c=0$, the equilibrium is $\left(K^{*}, L^{*}\right)=(0.9054,1)$ and there are no links between the dynamics of total population and the capital stock. Then, if $L$ converges to its stationary state value $L^{*}$, the dynamics of capital are driven by $\dot{K}=s A K_{\tau_{1}}^{\alpha}\left(L^{*}\right)^{1-\alpha}-\delta K_{\tau_{1}}$ so that fluctuations in $K$ can be obtained only for technological reasons [see Zak (1999) for a stability analysis of this case]. This is shown in Panel (a) of
Fig. 2 by setting $\tau_{1}=7.5$ and $\tau_{2}=0.48$. When $c=0$ and $\tau_{1}=0$, the opposite result holds so that fluctuations in $K$ are caused by fluctuations in $L$ [see Banks (1994) for a stability analysis of this case]. This is shown in Panel (b) of Fig. 2 plotted for $\tau_{2}=0.52$. By assuming now that both delays are positive and fixed at sufficiently large values $\left(\tau_{1}=7.5\right.$ and $\tau_{2}=0.507$ ), the fixed point of the system is unstable (This result can be ascertained through the use of the stability crossing curves technique.) and it is possible to identify a mutual relationship between the two dynamics. This phenomenon is well illustrated by the behaviour of the dynamics of $K$ (black coloured) in Panel (c) of Fig. 2, where short-term fluctuations (almost two years) sum up to long-term fluctuations (almost thirty years). Finally, by starting from this last 


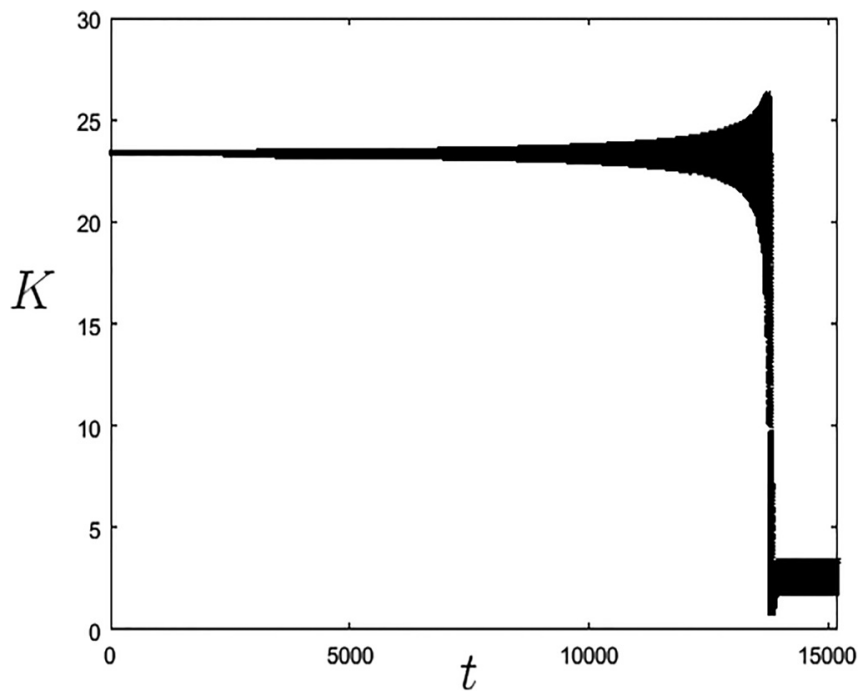

FIG. 4. Time series of $K$ for $\tau_{1}=10$ and $\tau_{2}=6.27$. The high equilibrium has just undergone a sub-critical Hopf bifurcation and, after a long transient characterised by oscillations around it, the dynamics are captured by the limit cycle around the low equilibrium.

case, the bifurcation diagram with bifurcation parameter $c$ plotted in Panel (d) shows how the feedback given by the production side of the economy can stabilise the system.

\section{B. Multiple steady-state equilibria}

In the previous paragraph, we have shown that parameter $c$ does not qualitatively affect the dynamics of the system when only one attractor exists and a change in it does not cause saddle node bifurcations. In this paragraph, instead, we shall consider a value of $c$ such that three equilibria do exist and show the role played by the delays in defining the dynamics of the system. For instance, by using the parameter set $s=0.3, A=0.9, \alpha=0.17$, $\delta=0.1, L_{0}=0.5, \bar{L}=7.74, \gamma=0.2$, and $c=0.215$, we obtain the equilibria $\left(K_{1}^{*}, L_{1}^{*}\right)=(2.95,0.89),\left(K_{2}^{*}, L_{2}^{*}\right)=(11.42,3.45)$, and $\left(K_{3}^{*}, L_{3}^{*}\right)=(23.39,7.07)$. This is shown in Panel (a) of Fig. 3, and Panels (b) and (c) depict the stability crossing

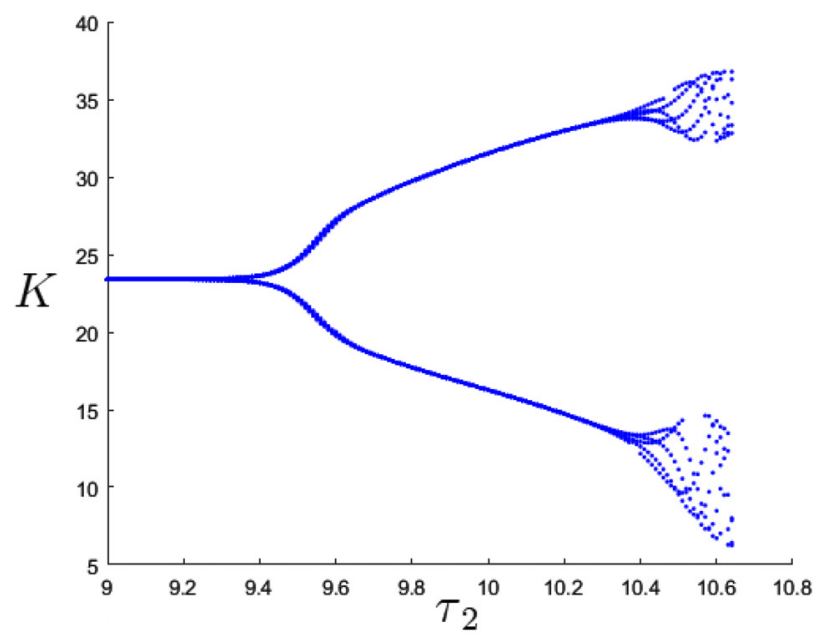

(a)

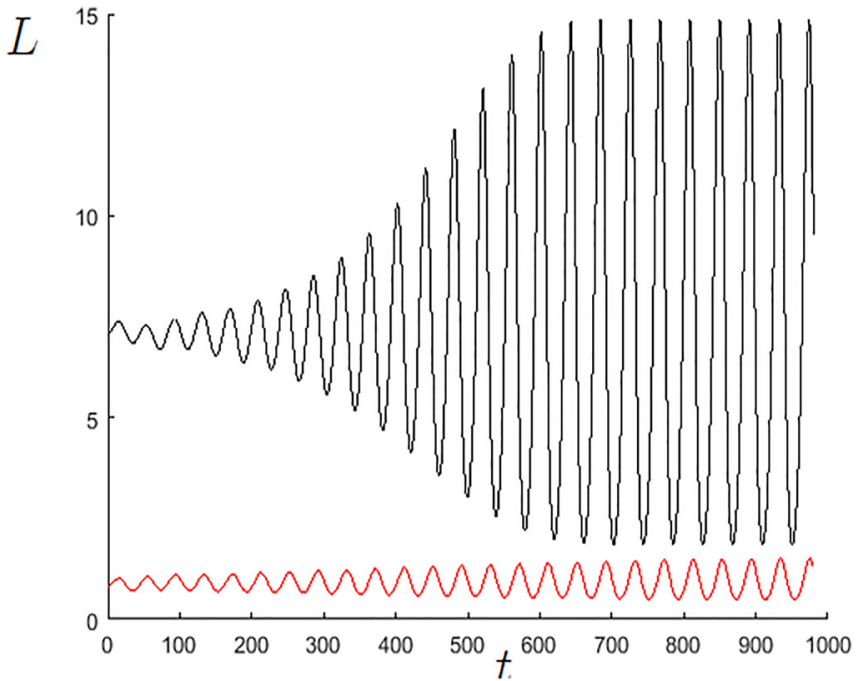

FIG. 5. Time series of $K$ for $\tau_{1}=30$ and $\tau_{2}=10$. Both equilibria have undergone a Hopf bifurcation and-depending on initial conditions - there are oscillations around one of the two equilibria. Initial conditions: $K(t)=24, L(t)=7$ for $t \in[-30,0]$ (black line); $K(t)=2.9, L(t)=0.8$ for $t \in[-30,0]$ (red line).

curves related to $\left(K_{1}^{*}, L_{1}^{*}\right)$ and $\left(K_{3}^{*}, L_{3}^{*}\right)$ in $\left(\tau_{1}, \tau_{2}\right)$ plane. In particular, the yellow and green regions represent, respectively, the areas in which $\left(K_{1}^{*}, L_{1}^{*}\right)$ and $\left(K_{3}^{*}, L_{3}^{*}\right)$ are stable, whereas the boundaries of these regions identify the points at which the stationary states generically (that is, if the transversality conditions are satisfied) undergo a Hopf bifurcation. In this model, identifying whether this bifurcation is supercritical or subcritical is difficult to be handled in a neat analytical form. Thus, we will tackle the problem numerically. First, by comparing the two equilibria $\left(K_{1}^{*}, L_{1}^{*}\right)$ and $\left(K_{3}^{*}, L_{3}^{*}\right)$, we note that for a given value of $\tau_{2}$ the latter loses stability for a lower value of $\tau_{1}$, as Figs. 3(b) and 3(c) clearly show. This means that in developed countries investments should provide shorter-term returns than in underdeveloped or developing countries to avoid instability. Differently, there are several switches between the position of the crossing curves defining the stability region with respect to $\tau_{2}$ in the two cases.

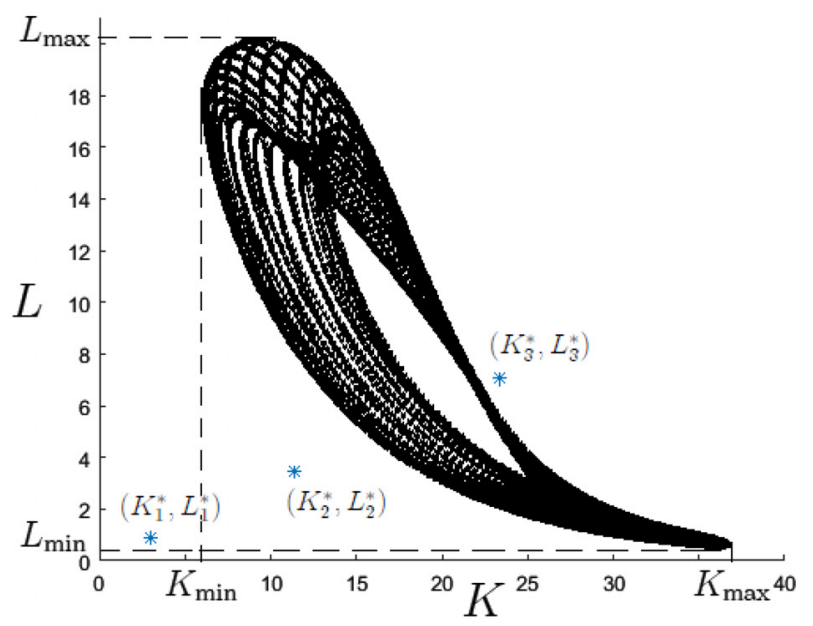

(b)

FIG. 6. (a) Bifurcation diagram with bifurcation parameter $\tau_{2}$. The diagram shows the maximum and minimum values taken by a typical trajectory that converges to the attractor. The increase in the number of points when $\tau_{2}$ becomes larger shows the increasing complexity of the system. (b) Projection of the attractor in the pseudo phase plane $(K, L)$ for $\tau_{1}=30$ and $\tau_{2}=10.5$. 
By referring to the parameter set used in the case of multiple steady-state equilibria, the simulation portrayed in Fig. 4 shows that trajectories are captured by the low equilibrium once the high equilibrium has been destabilised through a sub-critical Hopf bifurcation. This is an important result stressing the existence of a reversal towards underdevelopment (the poverty trap) caused by the presence of a time-tobuild technology from which there exists a delay from the time an investment is made to the time it becomes productive $\left(\tau_{1}\right)$ and delays from the time people were born to the time they will be economically active (belonging to the working population) and sexually active $\left(\tau_{2}\right)$. We note that an increase in the value of $\tau_{1}$ produces a change in the nature of the Hopf bifurcation (which becomes super-critical) undergone by the high equilibrium allowing the coexistence of limit cycles, as is shown in Fig. 5. However, we have verified that large fluctuations around the low equilibrium (induced by larger values of $\tau_{2}$ ) cause the death of the attractor and the high equilibrium just remains the unique attractor of the system so that trajectories are captured by the limit cycle around the high equilibrium. By using the same value of $\tau_{1}$ as in Fig. 5, Fig. 6(a) shows the evolution (bifurcation diagram) of the "high" attractor when $\tau_{2}$ varies. In particular, Fig. 6(b) depicts the attractor of the system for $\tau_{2}=10.5$ in the pseudo phase plane $(K, L)$. By increasing the value of $\tau_{2}$, it is possible to observe an enlargement of the attractor. To this purpose, Fig. 6(b) shows that the trajectories captured by the attractor are such that $L_{\max }>L_{3}^{*}, L_{\min }<L_{1}^{*}$ and $K_{\max }>K_{3}^{*}, K_{\min }<K_{2}^{*}$, where $X_{\max }$ and $X_{\min }$ are the maximum and minimum values of variable $X$, respectively.

\section{CONCLUSIONS}

This work analysed a Solow model augmented with a time-to-build technology and population dynamics employing carrying capacity constraints and time delays. The main aim is to characterise the existence of economic and demographic cycles and identify the chain of causation between the main variables. This result deserves a attention as it is not usual finding clear prediction about one-way or double feedback effects between economic and demographic variable in the neoclassical growth literature (ranging from models à la Solow to the more recent contributions belonging to the Unified Growth Theory). Then, the Solow model becomes able to understand the reasons why production and capital dynamics affect population dynamics or vice versa and therefore the reasons why some countries lie on trajectories leading to sustained development, whereas others remain entrapped in a Malthusian trap.

In the article, we assumed that the delays were fixed. However, a part of the scientific literature-especially the literature on demographic issues-has focused also on the study of models with distributed delays. This is to capture in a more appropriate way the role played by the population age structure (Fanti et al., 2013 and Beretta and Breda, 2016) and the existence of economies where production takes place through the use of some kinds of vintage technologies (Boucekkine et al., 2002). These issues will be accounted for in our future research agenda.

Banks, R. B., Growth and Diffusion Phenomena-Mathematical Frameworks and Applications (Springer-Verlag, NY, 1994).

Barro, R. J. and Sala-i-Martin, X., Economic Growth, 2nd ed. (MIT Press, Cambridge (MA), US, 2003).

Becker, G. S., "An economic analysis of fertility," in Demographic and Economic Change in Developing Countries, National Bureau Committee for Economic Research (Princeton University Press, Princeton (NJ), 1960).

Beretta, E. and Breda, D., "Discrete or distributed delay? Effects on stability of population growth," Math. Biosci. Eng. 13, 19-41 (2016).

Boucekkine, R., de la Croix, D., and Licandro, O., "Vintage human capital, demographic trends, and endogenous growth," J. Econ. Theory 104, 340-375 (2002).

Brock, W. A. and Taylor, S., "The Green Solow model," J. Econ. Growth 15, 127-153 (2010).

Cai, D., "Multiple equilibria and bifurcations in an economic growth model with endogenous carrying capacity," Int. J. Bifurcation Chaos Appl. Sci. Eng. 20, 3461-3472 (2010).

Cai, D., "An economic growth model with endogenous carrying capacity and demographic transition," Math. Comput. Modell. 55, 432-441 (2012).

Dalgaard, C.-J. and Strulik, H., "The history augmented Solow model," Eur. Econ. Rev. 63, 134-149 (2013).

Doepke, M., Hazan, M., and Maoz, Y. D., "The baby boom and World War II: A macroeconomic analysis,” Rev. Econ. Stud. 82, 1031-1073 (2015).

Ehrlich, I. and Lui, F., "The problem of population and growth: A review of the literature from Malthus to contemporary models of endogenous population and endogenous growth," J. Econ. Dyn. Control 21, 205-242 (1997).

Fanti, L. and Manfredi, P., "The Solow's model with endogenous population: A neoclassical growth cycle model," J. Econ. Dev. 28, 103-115 (2003).

Fanti, L., Iannelli, M., and Manfredi, P., "Neoclassical growth with endogenous age distribution. Poverty vs low-fertility traps," J. Popul. Econ. 26, 1457-1484 (2013).

Galor, O., Unified Growth Theory (Princeton University Press, Princeton (NJ), 2011).

Galor, O., "The demographic transition: Causes and consequences," Cliometrica 6, 1-28 (2012).

Galor, O. and Moav, O., "Natural selection and the origin of economic growth,” Q. J. Econ. 117, 1133-1191 (2002).

Galor, O. and Moav, O., "From physical to human capital accumulation: Inequality and the process of development," Rev. Econ. Stud. 71, 1001-1026 (2004)

Galor, O. and Weil, D. N., "Population, technology, and growth: From Malthusian stagnation to the demographic transition and beyond," Am. Econ. Rev. 90, 806-828 (2000).

Jones, L. E. and Schoonbroodt, A., "Baby busts and baby booms: The fertility response to shocks in dynastic models," Rev. Econ. Dyn. 22, 157-178 (2016).

Leibenstein, H. M., Economic Backwardness and Economic Growth (Wiley, New York (NY), 1957).

Lin, X. and Wang, H., "Stability analysis of delay differential equations with two discrete delays," Can. Appl. Math. Q. 20, 519-533 (2012).

Livi-Bacci, M., A Concise History of World Population, 6th ed. (WileyBlackwell, Malden, 2017).

Mankiw, N. G., Romer, D., and Weil, D. N., "A contribution to the empirics of economic growth,” Q. J. Econ. 107, 407-437 (1992).

Palivos, T., "Endogenous fertility, multiple growth paths, and economic convergence," J. Econ. Dyn. Control 19, 1489-1510 (1995).

Pecora, N. and Sodini, M., "A heterogeneous Cournot duopoly with delay dynamics: Hopf bifurcations and stability switching curves," Commun. Nonlinear Sci. Numer. Simul. 58, 36-46 (2018).

Roser, M. and Ortiz-Ospina, E., https://ourworldindata.org/world-population-growth for World Population Growth, 2018.

Solow, R. M., "A contribution to the theory of economic growth," Q. J. Econ. 70, 65-94 (1956).

Zak, P. J., "Kaleckian lags in general equilibrium," Rev. Polit. Econ. 11, 321-330 (1999) 